Proc. Indian Acad. Sci. (Chem. Sci.), Vol. 102, No. 5, October 1990, pp. 575-579.

(C) Printed in India.

\title{
Density of states of fractons on percolating networks
}

\author{
T NAKAYAMA* and K YAKUBO \\ Department of Applied Physics, Hokkaido University, Sapporo 060, Japan
}

\begin{abstract}
We have performed computer experiments on the dynamics of three-dimensional percolating networks. The results of the densities of states indicate the theoretically expected relation $D(\omega) \sim \omega^{1 / 3}$ for scalar displacements. We also present the results of the density of states of fractons in which the Hamiltonian incorporates both bond-stretching and bond-bending force constants.
\end{abstract}

Keywords. Three-dimensional percolating networks; density of state; fractons; scalar displacements.

Over the past few years vibrational modes on percolating networks have received a great deal of attention (Alexander and Orbach 1982; Rammal and Toulouse 1983; Courtens et al 1988; Tsujimi et al 1988). In particular, the scaling theory has made much progress in understanding these problems (Alexander and Orbach 1982; Rammal and Toulouse 1983). Percolating networks have a fractal geometry on smaller length-scales than the percolating correlation length $\xi_{p}$. This behaviour crosses over to the Euclidean geometry on larger scales than $\xi_{p}$. The vibrational density of states with wavelenghts much larger than $\xi_{p}$ should obey the conventional Debye law, $D(\omega) \sim \omega^{d-1}$, where $d$ is the Euclidean dimension.

The scaling arguments (Alexander and Orbach 1982; Rammal and Toulouse 1983) have suggested that the density of states (DOS) for fractal structures follows the universal law, $D(\omega) \sim \omega^{\overline{\bar{d}}-1}$, where $\overline{\bar{d}}$ is the fracton dimensionality. When percolating networks are characterized by the fractal dimension $D$ and the exponent of diffusion $\theta$, the fracton (spectral) dimension $\overline{\bar{d}}_{s}$ is expressed as $\overline{\bar{d}}_{s}=2 D /(2+\theta)$. Alexander and Orbach (1982) have conjectured that the fracton dimensionality $\overline{\bar{d}}_{s}$ for percolating network with scalar displacements is $4 / 3$, which is independent of the Euclidean dimension $d$. The conjecture was confirmed recently from light scattering experiments for aerogels (Courtens et al 1988; Tsujimi et al 1988). Though samples for these experiments are considered as mass-fractal materials, it is not obvious that vibrational excitations are due to only scalar displacements.

In this article, we have performed computer experiments on the dynamics of three-dimensional (3D) site-percolating elastic networks consisting of the site number, $N>10^{5}$ atoms. The results of the DOS's for scalar fractons on 3D-percolating networks show the theoretically expected relation $\overline{\bar{d}}_{s}=4 / 3$ and the existence of two distinct frequency regimes in the DOS characterized by $\omega_{c}$ which corresponds to the wavelength close to $\xi_{p}$. In addition, it is found that no notable steepness or hump

\footnotetext{
* For correspondence
} 
exists in the phonon-fracton crossover region in conflict with the effective-medium theory (Derrida et al 1984) and mode-counting consideration (Aharony et al 1985). We have also performed computer experiments for the DOS of vector fractons excited on 2D-percolating networks. The results indicate that the vector fracton dimensionality $\overline{\bar{d}}_{v} \simeq 0 \cdot 9 \pm 0 \cdot 1$.

We consider site-percolating networks consisting of $N$ atoms with mass $m$ and linear springs connecting two nearest-neighbor atoms. The equations of motion of the atoms are

$$
m \ddot{u}_{i}-\sum_{j} K_{i j} u_{j}=0
$$

where $u_{i}$ is the displacement of the atom on the ith site. The force constant is taken to be $K_{i j}=0(i \neq j)$ if either sites $i$ or $j$ are unoccupied, and $K_{i j}=1$ otherwise. Diagonal elements satisfy the following relation $K_{i i}=-z_{i}$, where $z_{i}$ is the coordination number of the site $i$. The displacement $u_{i}$ has only one component. This means that we treat the excitations with scalar displacements. The numerical technique is based on an idea that the eigenfrequency of the system satisfies the resonance condition when applying the periodic external force with frequency $\omega$ to the system (Williams and Maris 1985). The method has the following advantages; (i) one can apply this algorithm to a very large system $\left(N>10^{5}\right)$. Specifically, the numerical accuracy increases with increase in site number; (ii) it is possible to calculate quite accurately the DOS in the low frequency regime; (iii) the magnitudes of the DOS at any frequencies can be obtained by sweeping the resonant frequency; (iv) the algorithm is extraordinarily suitable for array-processing supercomputers.

The accuracy of the algorithm was checked by comparing the DOS obtained for the regular $2 \mathrm{D}$-square lattice $(100 \times 100)$ and $3 \mathrm{D}$-cubic lattice $(30 \times 30 \times 30)$ with the corresponding exact solutions. The numerical results agreed well with the exact solutions. Especially in the very low-frequency regime the agreement was excellent.

The DOS of a 3D site-percolating network at $p=0.4\left(p_{c}=0.312\right)$ formed on a $70 \times 70 \times 70$ simple cubic lattice is shown in figure 1 . The network size is $N=122,448$. We can see that the DOS in the frequency region $0 \cdot 1<\omega<1$ is proportional to $\omega^{1 / 3}$, that is, the Alexander-Orbach conjecture holds for the 3D case. The DOS in the low frequency regime $(\omega \ll 0 \cdot 1)$ obeys the Debye's law $D(\omega) \sim \omega^{2}$ where the phonon-fracton crossover frequency $\omega_{c}$ is close to $0 \cdot 1$. Note here that the wavelength corresponding to $\omega_{c}$ is related to the percolation correlation length $\xi_{p}$ varied as $\left|p-p_{c}\right|^{-v}$, where the critical exponent $v$ is a positive constant. The system can be regarded as a homogeneous continuum on larger scales than $\xi_{p}$, so our numerical result in the low frequency regime is reasonable. Figure 1 tells us that the DOS is smoothly connected from the phonon regime to the fracton regime. Especially, in contrast to the prediction of the effective-medium theory (Derrida et al 1984), our result does not exhibit a notable steepness or the presence of the hump in the vicinity of $\omega_{c}$. The DOS does not follow the $\omega^{1 / 3}$ dependence above $\omega \sim 1$. This is due to the fact that the system is not fractal on a length scale shorter than the wavelength corresponding to $\omega \sim 1$.

Some experimental results for topologically disordered materials indicate the existence of "steepness" or "hump" in the crossover region of the DOS (Rosenberg 1985). However, the low-frequency inelastic light scattering experiment for superionic borate glass with the fractal structure shows no steepness, nor does it show the hump in the crossover region (Fontana et al 1987). Furthermore, the Brillouin scatterings 


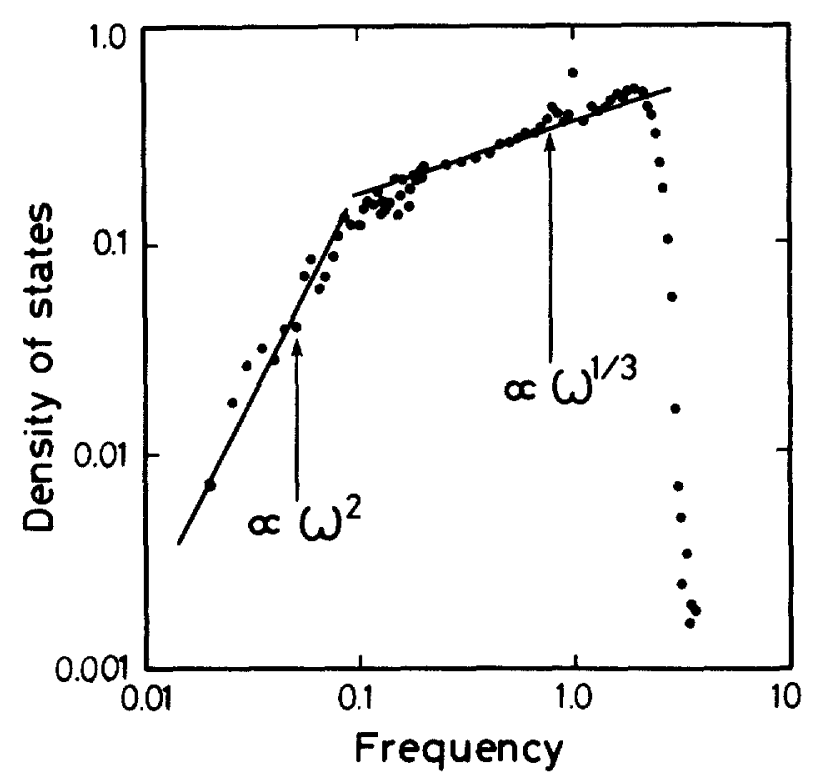

Figure 1. The DOS of fractons with scalar displacements excited on a 3D site-percolating network at $p=0 \cdot 4$. The network is formed on a $70 \times 70 \times 70$ simple-cubic lattice and has 122,448 atoms.

on a series of silica aerogels have revealed that the phonon-fracton crossover is characterized by smooth dispersion and less curves (Courtens et al 1988). It should be noted that vibrations in these systems are due to vector displacements rather than scalar ones.

We present the DOS of fractons excited on elastic percolating networks with vector displacements. The Hamiltonian is expressed by incorporating both bond-stretching $(\alpha)$ and bond-bending $(\beta)$ force constants:

$$
H=\frac{1}{2} \alpha \sum_{\langle i j\rangle} K_{i j}\left[\left(\mathbf{u}_{i}-\mathbf{u}_{j}\right) \cdot \mathbf{r}_{i j}\right]^{2}+\frac{1}{2} \beta \sum_{\langle i j k\rangle} K_{i j} K_{j k}\left(\delta \theta_{i j k}\right)^{2} .
$$

Here $\mathbf{u}_{i}$ is the vector displacement of the atom $i, \mathbf{r}_{i j}$ the directional vector of the nearest neighbors $\langle i j\rangle, \delta \theta_{i j k}$ the change in the angle between the bond $\langle i j\rangle$ and $\langle j k\rangle$ due to displacements of these atoms. $K_{i j}$ has the same meaning as that in (1). $\alpha$ and $\beta$ represent the strength of the bond-stretching and bond-bending elastic force constant, respectively. This vector-displacement model describes vibrational excitations in actual amorphous solids. It has been suggested that the universality class of the vector model is different from that of the scalar model (Kantor and Webman 1984). Accordingly, the fracton dimensionality $\bar{d}_{v}$ for vector fractons has a different value from $\bar{d}_{s}$ (Feng 1985 ; Webman and Grest 1985). Figure 2 shows the DOS for the 2D-percolating network described by (2), where the percolating network at $p_{c}$ contains the site number of 8,155 atoms. Filled and open circles indicate the DOS for the force constant ratio $\beta / \alpha=0.01$ and $0 \cdot 1$, respectively. We see from this figure that the DOS diverge weakly with values approaching $\omega=0$. It is suggested by the scaling arguments that excitations in the lower frequency regime behave as vector fractons (Feng 1985). Our results indicate that the vector fracton dimensionally $\overline{\bar{d}}_{v}$ takes the value $0.9 \pm 0.1$, which was obtained 


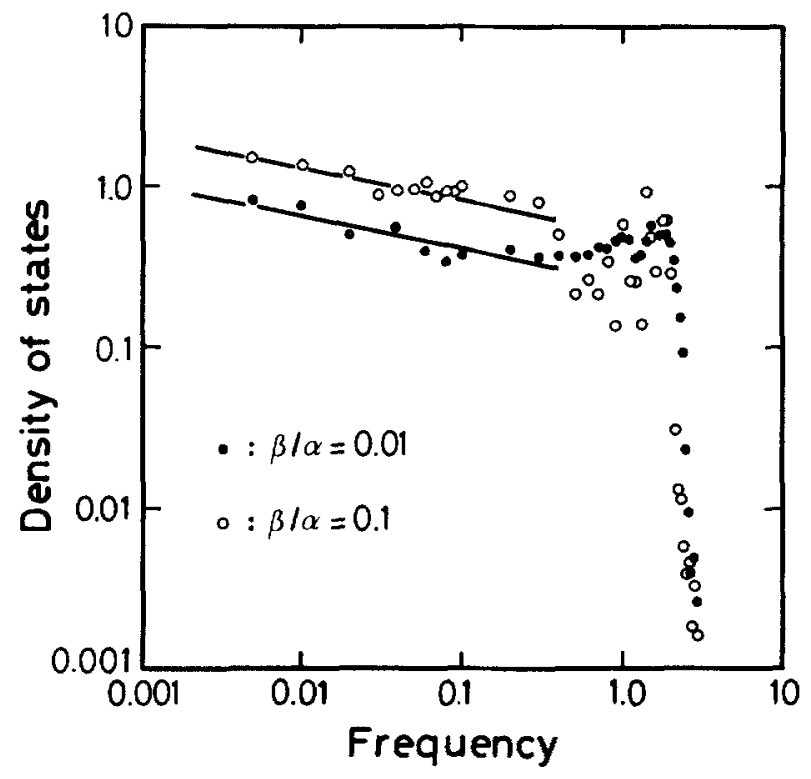

Figure 2. The DOS of fractons with vector displacements. The sample was formed on a $200 \times 200$ square lattice and has 8,155 atoms. The solid lines are proportional to $\omega^{-0 \cdot 2}$.

from the least square fitting for calculated data in the lower frequency regime. This value agrees, within numerical accuracy, with the theoretical prediction (Webman and Grest 1985) based on the nodes-links-blobs model. The detailed results for vector fractons will be published elsewhere.

We have performed computer experiments on the fracton dynamics of percolating networks with large particle size $\left(N>10^{5}\right)$. In the vicinity of the crossover between phonons and fractons, the DOS is smoothly connected (figure 1) and the results do not exhibit a notable steepness or a hump. Our numerical results of the DOS correctly show that the integrated number of modes equals the degree of freedom. It seems that the absence of the hump in the vicinity of the crossover region implies the appearance of excess density of states in the phonon regime. The DOS of vector fractons have been also presented. The value of $\overline{\bar{d}}_{v}$ agrees with the theoretical prediction. The details of this work will be published in future.

\section{Acknowledgement}

The Hokkaido University Computing Center is acknowledged for the use of the supercomputer facilities.

\section{References}

Aharony A, Alexander S, Entin-Wohlman O and Orbach R 1985 Phys. Rev. B31 2565

Alexander S and Orbach R 1982 J. Phys. (Paris) Lett. 43 L625

Courtens E, Vacher R, Pelous J and Woignier T 1988 Europhys. Lett. 6245 
Derrida B, Orbach R and Yu K W 1984 Phys. Rev. B29 6645

Feng S 1985 Phys. Rev. B32 5793

Fontana A, Rocca F and Fontana M P 1987 Phys. Rev. Lett. 58503

Kantor Y and Webman I 1984 Phys. Rev. Lett. 521891

Rammal R and Toulouse G 1983 J. Phys. (Paris) Lett. 44 L13

Rosenberg H M 1985 Phys. Rev. Lett. 54704

Tsujimi Y, Courtens E, Pelous J and Vacher R 1988 Phys. Rev. Lett. 602757

Webman I and Grest G S 1985 Phys. Rev. B31 1689

Williams M L and Maris H J 1985 Phys. Rev. B31 4508 